\title{
INFORMACION
}

\section{Séptimo Congreso Internacional de Literatura Iberoamericana}

\author{
Resoluciones
}

El doctor Antonio Castro Leal, en nombre de la Universidad Nacional Autónoma de México, propuso la siguiente iniciativa:

La Universidad Nacional de México ofreció al Instituto Internacional de Literatura Iberoamericana publicar en las prensas universitarias mexicanas una serie de clásicos de América, en ediciones preparadas por los miembros del Instituto.

Diversas circunstancias, entre ellas la falta de un plan concreto de trabajo, impidieron la realización del proyecto. La Universidad de México sigue interesada en la idea de difundir el conocimiento de la literatura iberoamericana por medio de ediciones apropiadas, y desea renovar ahora su invitación anterior proponiendo publicar comentarios y textos relativos al período de 1900 a 1950. La colección constaría:

1. De panoramas o historias de la literatura de algún país iberoamericano, o de algún aspecto de su literatura (poesía, novela, teatro, etc.), durante 1900-1950;

2. Antologías de los diversos géneros literarios (poesía, novela, teatro, ensayo, cuentos, etc.), que llevarían un prólogo explicativo y notas biobibliográficas y críticas de los autores incluídos;

3. Aunque es indudable que los trabajos tendrían una extensión variable, sería conveniente uniformarlos dentro de 
dos categorías: el volumen normal y el volumen de doble extensión;

4. Para fijar la extensión del volumen normal habria que pensar en el material indispensable para presentar a las figuras de primera, segunda y acaso tercera categoría que representaran el período, o alguno de los aspectos del período escogido;

5. Aunque la serie se refiere al período $1900-1950$ podrían rebasarse estas fechas en algunos años, cuando fuera necesario para explicar antecedentes o continuaciones de ciertos movimientos;

6. La Universidad de México se encargará de la edición y distribución de la serie, así como del pago de los honorarios a los miembros del Instituto que preparen los volúmenes de ella, de acuerdo con la tarifa acostumbrada de $10 \%$ sobre el precio al público de la edición total, y

7. De los productos de la venta se resarciría la Universidad de México de los gastos hechos y el sobrante serviría para desarrollar el programa editorial del Instituto.

La Comisión de Inciativas propone el siguiente acuerdo: Procédase a la publicación de esa biblioteca y desígnese director de la misma al doctor Antonio Castro Leal, a fin de que, de acuerdo con la Mesa Directiva, realice las gestiones necesarias para la edición de las obras. [Aprobada.]

El ser̃or Raúl Silva Castro propuso la siguiente iniciativa:

Teniendo presente la conveniencia de acelerar los estudios regionales de literatura o la creación de los mismos, conforme los siguientes principios:

I. E1 Instituto debe funcionar en un establecimiento de enseñanza superior que le acredite la independencia necésaria para la realización de sus fines, o adscrito a la Biblioteca Nacional del país respectivo, según sea el caso;

II. El Instituto debe propender a la formación de archivos sistemáticos del material adecuado para el estudio de la literatura del país correspondiente, así como para el debido conocimiento de las influencias que se hayan recibido de otros países;

III. El Instituto debe dar cuenta periódicamente de los resultados de sus investigaciones, en un boletín o publicación de carácter similar, que contenga en lo posible la bibliografía nacional selecta que interesa a la investigación literaria. 
La Conferencia, en fin, recomienda adscribir a los institutos nacionales de literatura, a título honorífico o con remuneración, a críticos literarios, bibliógrafos, historiadores de la literatura, catedráticos del ramo, etc., con la mayor amplitud posible, a fin de que la obra del establecimiento se distinga por su imparcialidad, su tolerancia y su espíritu de justicia.

La Comisión de Inciativas propone el siguiente acuerdo: Amplíense los estatutos del Instituto Internacional de Literatura Iberoamericana con el siguiente agregado a su artículo primero, inciso " $\mathrm{k}$ ": Recomendar asimismo la creación de Institutos de Literatura que estén formados por los profesores y especialistas en la materia y que puedan disponer de los medios necesarios para el mejor cumplimiento de su propósito. [Aprobada.]

E1 doctor George O. Schanzer propuso la siguiente iniciativa relativa a la enseñanza de las letras iberoamericanas en los Estados Unidos :

A. The Institute will appoint a committes of scholars, headed by a man of prominence, to survey the present state of teaching of Latin American Literature in the U. S.

$B$. The actual survey will be the work of one man who undertakes the task on leave from his own institution, which will sponsor his project for a Ford Grant. With the backing of his University and the encouragement of the Institute, the young scholar is most likely to receive the grant for $1957 / 58$.

C. The study will include the teaching of the subject on all levels. It will examine its place in the curriculum of major, minor, education, and area students. It ought to gauge its place in relation to peninsular studies. It should not be limited to circulars, but should inchde personal contact and observation.

D. The surveyor will be guided and aided by the Institute's Committee which will receive, edit, and publish his report and make suitable recomendations.

E. The study may include the past and present use of texts and other works on Spanish American Literature -including those published under the auspices of the Institute- in the teaching of the subject, with a view to their possible revision or replacement.

F. The Institute may then undertake to re-edit its texts, which have served us so well, on the basis of collective recommendations. 
La Comisión de Iniciativas propone pasar la ponencia a la Mesa Directiva, a fin de que estudie la conveniencia de realizar to propuesto en la iniciativa. En caso afirmativo, que dicha Mesa disponga dar los pasos necesarios para tal fin.

IV

El profesor Alfredo Angel Roggiano propuso la siguiente iniciativa:

"En virtud de que en el año próximo - 1956- se cumplen diez años de la muerte del doctor Pedro Henríquez Ureña, la Comisión de Iniciativas recomienda por unanimidad la dedicación de un número de la Revista Iberoamericana, correspondiente a dicho año, a comentar la obra del ilustre humanista de la América Hispánica, y que se dirija a las agrupaciones culturales para que rindan homenajes semejantes en Hispanoamérica." [Aprobada].

$\mathrm{V}$

E1 VII Congreso del Instituto Internacional de Literatura Iberoamericana agradece a la Universidad de California su hospitalidad y las muchas atenciones dispensadas a sus miembros durante el desarrollo del Congreso, y pide para la Universidad y su Canciller un aplauso. [Aprobada.]

VI

E1 VII Congreso del Instituto Internacional de Literatura Iberoamericana hace constar su agradecimiento al Secretario Ejecutivo del Instituto, profesor Marshall R. Nason, por la actividad que ha desplegado en sus labores al servicio del Instituto y para el mejor éxito del Congreso, y pide para él un aplauso. [Aprobada.]

VII

E1 VII Congreso agradece a los Comités locales la actividad, celo y dedicación con que han contribuído al éxito de este Congreso, integrados de la siguiente manera: Comisión de Trabajos: Luis Monguió, Presidente; Edwin S. Morby, Fernando Alegría y Benjamín Woodbridge, miembros; Comité de Recepción: Robert Anderson, Presidente; James Holton, Paul Lloyd, Bárbara Mortenson y Robert Page, miembros; Comité de Banquete: Edwin J. Webber, Presidente; E. Hortense White y Marian Fredine, miembros. Se pide un aplauso para ellos. [Aprobada.]

VIII

El VII Congreso aprueba la gestión de la Mesa Directiva saliente, la felicita por el feliz cumplimiento de sus labores y pide para ella un aplauso. [Aprobada.] 


\section{Lista de delegados al Séptimo Congreso}

Alegría, Fernando. University of California.

Alten, Martha E. Mills College, Calif.

Anderson, Robert. University of California.

ANDERSON IMBERT, Enrique. University of Michigan.

Arce, José M. Dartmouth College.

Atrinson, William C. University of Glasgow.

Avila, Pablo. Santa Barbara College, U. C.

Austin, Brother. St. Mary's College.

Ayala, Francisco. Universidad de Puerto Rico.

Bary, David. Universidad de California.

BERRIEN, William. University of Southern California.

Benton, Gabriela von Munk. Occidental College.

Bramage, Julia A. Santa Barbara Junior College.

Castro Leal, Antonio. Mexico City College.

Clarke, Dorothy Clotelle. University of California.

Corbató, Hermenegildo. U. C. L. A.

Correa, Gustavo. University of Chicago.

Charouhas, T. G. University of California.

Davison, Ned J. University of Oregon.

Deuel, Pauline B. University of Redlands.

Duncan, R. M. Universidad de Nuevo México.

Ezcurdia, Manuel de. University of California.
Fajardo, Raúl José. San Carlos, California.

Ferrer Canales, José. Dillard University.

Fisher, Vera P. Coachella Va1ley. Union High School.

Fogelguist, Donald F. U. C. L. A.

García-Girón, Edmundo. Universidad de Oregon.

Glickman, Robert Jay. U. C. L. A.

Gaw, Esther Allen. Berkeley, California.

Grcovate, Bernardo. Tulane University.

Holton, James S. Sacramento State College.

Henríluez Ureña, Max. University of California, L. A.

Hannan, Denis. Southern Oregon College.

Hulet, Claude L. Washington University.

Inostroza, Raúl. Army Language School, Monterey, Calif.

Jaimes Freyre, Mireya. University of California.

Ham MOND, George P. University of California.

García, Bárbara M. Mills College.

Hilton, Ronald. Stanford University.

Jiménez Rueda, Julio. Universidad Nacional de México.

Keller, Daniel S. University of California, Davis.

Kirschenbaum, Leo. U. C. L. A.

Lamb, Ruth S. Scripps College, Calif.

Levy, Kurt L. University of Toronto. 
Malle, Jerónimo. University of Iowa.

Marín, Juan. Santiago de Chile.

McCullough, J. T. Sacramento State College.

Mimrane, Freda. Dominican College of San Rafael.

Monguió, Luis. Mills College.

Monterde, Francisco. Universidad Nacional de México.

Moellering, William. San José State College.

Mosk Sanford, A. University of California.

Morby, Edwin S. University of California.

Mortenson, Bárbara. University of California.

Millán, María del Carmen. Universidad Nacional de México.

Mrsuraca, Sonia. Upland, California.

Moser, Gerald M. Pennsylvania State College.

Nason Marshall, R. University of New Mexico.

Nelken, Zoila. Ross, California.

Ortigoza, Carlos. University of Colorado.

OsGood, Robert S. Chula Vista, California.

Parker, Jack H. Universidad de Toronto.

Perales Ojeda, Alicia. Universidad Nacional de México.

Reyes, José Luis. San Francisco State College.

Rivas, Enrique de. University of California.

Roberts Graves, B. Southwestern Louisiana Institute.

Roberts, William Holloway. Vanderbilt University. Nashville, Tenn.
Rodríguez Alcalá, Hugo. Washington State College.

Rodríguez, Mario B. University of Arizona.

Roggiano, Alfredo Angel. State University of Iowa.

Rodríguez-Díaz, M. Alfred University.

Ross, Cecilia. University of California.

Schade, George D. University of Texas. Austin, Texas.

Schanzer, George O. St. John's University.

Schulman, Ivan A. U. C. L. A.

Shirley, Joseph Chilton. Stanford University.

Spell, J. R. University of Texas, Austin.

Swain, James O. University of Tennessee, Knoxville.

Terán, Carlos M. Michigan State University.

Torre, Manolo de la. Cuba.

Torres-Ríoseco, Arturo. University of California.

Torres Anderson, Grace. San Francisco, California.

Trejo, Arnulfo. U. C. L. A.

VAN Horne, John. University of Illinois. Urbana, Illinois.

Vázquez Amaral, José Francisco. Rutgers University, New Jersey.

Very, Francis G. University of California.

WebBer, Edwin J. University of California.

Williams, May E. Los Angeles, California.

Wilson, Irma. Arizona State College at Tempe.

ZEIDNER, Betty. University of California. 